\title{
Long-Term Safety and Efficacy of Fecal Microbiota Transplantation in the Treatment of Clostridium difficile Infection in Patients With and Without Inflammatory Bowel Disease: A Tertiary Care Center's Experience
}

\author{
Obada M. Tabbaa ${ }^{\mathrm{a}, \mathrm{c}}$, Mohammed M. Aboelsoud ${ }^{\mathrm{b}}$, Mark C. Mattar ${ }^{\mathrm{b}}$
}

\begin{abstract}
Background: Clostridium difficile infection (CDI) carries a large burden on the national public health with its high morbidity and mortality rates. Patients with inflammatory bowel disease (IBD) are generally at higher risk of infection, recurrence and complications. Therefore, the need for more reliable and safe therapy is necessary. Our study aims to evaluate long-term fecal microbiota transplant (FMT) outcomes in the general population compared to patients with IBD.
\end{abstract}

Methods: A single center long-term follow-up study was conducted to evaluate the outcomes of FMT in patients with and without IBD. Prior to FMT data including demographics, prior treatment of CDI and severity of symptoms were gathered via chart review. Post FMT, all patients were surveyed after 2 days, 30 days and $>1$ year to assess clinical and laboratory response. Our study outcomes included primary cure rate (negative CDI testing $>1$ year after single FMT), and secondary cure rate (negative CDI testing $>1$ year after repeat FMT or after an additional course of antibiotic with or without repeat FMT).

Results: Seventy-eight patients with recurrent or refractory CDI and subsequent FMT treatment were included. Mean age was 57 years, and $69 \%$ were females and twenty-one $(27 \%)$ had IBD. Primary cure rate was achieved in $77 \%$ of the cases while secondary cure rate reached $100 \%$ at the end of the study. IBD patients were younger with an average age of 47 years, and had more complains of abdominal pain $(71 \%)$, and required escalation of therapy in $50 \%$ of patients.

Conclusions: FMT was effective in the eradication of CDI in patients with and without IBD, but with no significant symptoms improvement in patients with IBD. Future randomized control studies

Manuscript submitted September 16, 2018, accepted October 8, 2018

aDepartment of Medicine, Medstar Washington Hospital Center, Washington, DC 20010, USA

${ }^{b}$ Division of Gastroenterology and Hepatology, Medstar Georgetown University Hospital, Washington, DC 20007, USA

${ }^{\mathrm{c} C o r r e s p o n d i n g ~ A u t h o r: ~ O b a d a ~ M . ~ T a b b a a, ~ D e p a r t m e n t ~ o f ~ M e d i c i n e, ~ M e d s t a r ~}$ Washington Hospital Center, 110 Irving St NW, Washington, DC 20010, USA. Email: Obada.M.Tabbaa@medstar.net

doi: https://doi.org/10.14740/gr1091 are needed to examine the long-term progression of IBD and quality of life in patients treated with FMT compared to standard therapy of antibiotics for recurrent CDI.

Keywords: Fecal microbiota transplant; Clostridium difficile; IBD; Outcomes

\section{Introduction}

Clostridium difficile infection (CDI) carries a high national public health burden, with infection rates reaching 147.2 cases/100,000 persons [1], accompanied by high morbidity and mortality $[2,3]$. CDI also has a high risk of recurrence, with approximately $10 \%-30 \%$ of patients developing at least one recurrent CDI episode, and risk increases with each successive infection $[4,5]$. More problematic is the tendency of CDI to affect vulnerable populations including the elderly, immunocompromised and patients with inflammatory bowel disease (IBD) [6-8].

Patients with IBD have been found to have higher rates of recurrence of CDI [8]. They are also at increased risk of having worse outcomes of CDI, including higher rates of colectomy and death [9]. Several factors have been identified as major contributors to the etiology of IBD: the activation and alteration of the mucosal immune system (loss of immune tolerance) [10]. A decrease in microbial biodiversity has been found in the mucosa and feces of IBD patients [11]. Therefore, the gut microbiome has been proposed as a key driver in the immune response seen in IBD and has gained more focus in recent observational studies [12].

Several proposed factors play crucial role in the association between IBD and CDI including: drugs that are used for the treatment of IBD, which could alter the intestinal flora and promote colonization (including repeat courses of antibiotics), altered immune and nutritional status, frequent hospitalizations and even genetic predisposition $[13,14]$. Thus, routine screening for CDI is recommended in all hospitalized IBD patients with disease flare-ups, according to the American College of Gastroenterology (ACG) [15]. It also recommends against escalation of immunosuppression therapy in patients with CDI, thereby posing a significant limitation in the treat- 
ment of disease flare-ups and inducing remission.

All these factors have led to increased interest in fecal microbiota transplantation (FMT), as a safe, efficacious and cost-effective alternative to recurrent or continuous courses of antibiotic therapy [16]. With a success rate averaging nearly $92 \%$ [17], FMT has been incorporated in 2018 Infectious Disease Society of America (IDSA) guidelines for the treatment of the second recurrence of CDI [18]. The absence of clear defining guidelines on treatment of CDI in IBD patients makes it more challenging. The available literature regarding CDI and IBD is mixed and limited on the long-term safety and efficacy of FMT in IBD patients. Some studies have showed FMT as less effective treatment for this population [19], and disease flare-ups have been reported following FMT in IBD patients [19-21]. Interestingly, two recent randomized controlled trials evaluating FMT in ulcerative colitis (UC) came to opposite conclusions. But both were underpowered to detect significant differences between FMT and placebo [22, 23].

Our single center study aims to enrich the limited data on long-term safety and effective profile of FMT in patients with IBD, along with those without IBD.

\section{Materials and Methods}

\section{Study design}

This is a retrospective observational, single institution study, conducted at the Division of Gastroenterology, MedStar Georgetown University Hospital, and was approved by the institutional review boards. We sought to evaluate the long-term safety and efficacy of FMT for recurrent/refractory CDI in patients with and without with IBD.

\section{Patient population}

We included adult patients ( $\geq 18$ years) who had recurrent or refractory CDI and underwent FMT between December 2012 and March 2017. CDI was defined as having diarrheal illness with positive stool $C$ difficile EIA or PCR test. Recurrent CDI was defined as more than one episode of CDI in 1 year. Refractory CDI was defined first CDI episode, which did not resolve within 7 days of standard antimicrobial treatment. Patients with severe CDI requiring ICU admission were excluded.

Demographic data, comorbidities, the presence of IBD and prior treatment for CDI were recorded for each patient. Severity of diarrhea was scaled as follows: mild $(<3$ bowel movements/day), moderate (3 - 6 bowel movements/day), or severe ( $>6$ bowel movements/day). For patients with IBD, baseline disease activity, prior IBD complications and current IBD treatment were recorded.

\section{FMT procedure}

The source of donor stool was obtained from OpenBiome stool bank or patients' relatives/friends when available. The method of delivery and the amount of stool delivered were decided upon on a case-by-case basis and at the discretion of the primary investigator. Methods of delivery included colonoscopy, flexible sigmoidoscopy, enema administration, push enteroscopy or through enteral feeding tubes.

Post FMT, all patients were surveyed after 2 days, 90 days and at $\geq 1$ year to assess the presence of diarrhea and severity, night time bowel movements, abdominal pain after FMT, use of antibiotics, hospitalizations related to diarrhea or abdominal pain, recurrence of CDI, development of new disease, and finally if they would consider FMT as their first choice if CDI happened to recur. Additional information collected for IBD patients post FMT included: subjective qualitative change in symptoms of diarrhea, abdominal pain, disease flare-up and escalation of therapy for IBD.

All patients were tested 2 weeks after FMT with repeat stool $C$ difficile EIA or PCR test. Early and late recurrences were defined as diarrheal illness plus positive testing within or after 90 days post FMT respectively.

\section{Study outcomes}

The main outcome of this study was to evaluate the efficacy and the safety of FMT through calculating the primary and the secondary cure rates. Primary cure was defined as diarrheafree and negative stool $C$ difficile testing results for $\geq 1$ year after single FMT. Secondary cure was defined as resolution of CDI after FMT with either another course of antibiotics or repeat FMT. As a secondary outcome, we compared the efficacy of FMT in patients with CDI who has IBD vs. not.

\section{Statistical analysis}

Statistical analyses were performed to evaluate the difference in demographics, symptoms severity, methods of delivery and types of antibiotics in patients with and without IBD. Continuous variables were described by means and standard deviations and two sample $t$-test and Wilcoxon rank sum (when normality assumption of the data was not satisfied (skewed data))tests as appropriate were used to compare distribution of continuous variables. Categorical variables were described by frequencies and percentages and Chi-square and Fisher exact (when cells have counts less than 5) tests as appropriate were used to compare proportions of categorical variables. Statistical significance was defined as $\mathrm{P}<0.05$.

\section{Results}

\section{Patients' characteristics and pre-FMT data}

Between 2012 and 2017, 83 FMTs were conducted, 78 patients were included in this study, and five patients were excluded for severe CDI. The mean age of study patients was $57.5 \pm$ 20.2 years, majority were females 69\% (54/78) and had FMT done in outpatient settings $87.2 \%$ (68/78). Severity of diarrhea 
Table 1. Characteristics of Patients With and Without IBD

\begin{tabular}{|c|c|c|}
\hline & IBD $(n=21)$ & Non-IBD $(n=57)$ \\
\hline Age in years, mean (SD) & $47.6(20.7)^{*}$ & $61.2(19.3)^{*}$ \\
\hline \multicolumn{3}{|l|}{ Gender n (\%) } \\
\hline Female & $9(42.9)^{*}$ & $45(79.0)^{*}$ \\
\hline Male & $12(57.1)^{*}$ & $12(21.1)^{*}$ \\
\hline \multicolumn{3}{|l|}{ FMT settings n (\%) } \\
\hline Inpatient & $4(19.1)$ & $6(10.5)$ \\
\hline Outpatient & $17(81.0)$ & $51(89.5)$ \\
\hline Abdominal pain n (\%) & $15(71.4)^{*}$ & $26(45.6)^{*}$ \\
\hline \multicolumn{3}{|l|}{ Severity of diarrhea n (\%) } \\
\hline Mild & $0(0.0)$ & $7(12.3)$ \\
\hline Moderate & $15(71.4)$ & $38(66.7)$ \\
\hline Severe & $6(28.6)$ & $12(21.1)$ \\
\hline \multicolumn{3}{|l|}{ Source of stool n (\%) } \\
\hline First degree relative & $7(33.3)$ & $21(36.8)$ \\
\hline OpenBiome & $13(61.9)$ & $34(59.7)$ \\
\hline Friend & $1(4.8)$ & $1(1.8)$ \\
\hline Med student & $0(0.0)$ & $1(1.8)$ \\
\hline \multicolumn{3}{|l|}{ Method of delivery n (\%) } \\
\hline CLN to TI & $18(85.7)$ & $44(77.2)$ \\
\hline CLN to cecum & $1(4.8)$ & $5(8.8)$ \\
\hline Throughout colon & $0(0.0)$ & $1(1.8)$ \\
\hline Flex sigmoidoscopy & $1(4.8)$ & $2(3.5)$ \\
\hline Enema & $0(0.0)$ & $1(1.8)$ \\
\hline $\mathrm{G} / \mathrm{J}$ tube & $0(0.0)$ & $2(3.5)$ \\
\hline Enteroscopy to jejunum & $0(0.0)$ & $1(1.8)$ \\
\hline \multicolumn{3}{|l|}{ Antibiotics used n (\%) } \\
\hline PO metronidazole & $15(71.4)$ & $40(70.2)$ \\
\hline PO vancomycin & $21(100.0)$ & $52(91.2)$ \\
\hline IV vancomycin & $3(14.3)$ & $5(8.8)$ \\
\hline Fidaxomycin & $7(33.3)$ & $15(26.3)$ \\
\hline
\end{tabular}

${ }^{*} \mathrm{P}<0.05$. FMT: fecal microbiota transplant; CLN: colonoscopy; TI: terminal ileum; G/J: gastrostomy and/or jejunostomy.

was described as moderate in $68 \%(53 / 78)$ of cases. Abdominal pain was present in $51 \%(41 / 78)$. All except four patients $93.6 \%(73 / 78)$ had at least one course of vancomycin. Out of the 78 patients, 21 had IBD (13 patients had UC and eight had Crohn's). The IBD subpopulation were younger (mean age $47.6 \pm 20.7$ vs. $61.2 \pm 19.3$ years $)$, mainly males $(57.1 \%$ vs. $21.1 \%)$ and reported more abdominal pain $(71.4 \%$ vs. $45.6 \%)$ when compared to non-IBD patients. There was no difference in the severity of diarrhea, prior antibiotic use, source of stool or method of delivery. Table 1 summarizes patients' characteristics in FMT patients with and without IBD. At the time of FMT, IBD patients were taking the following medications alone or in combination: ASA $76 \%$ (16/21), steroids $33.3 \%$
(7/21), anti-TNF 38\% (8/21), immunomodulators 24\% $(5 / 21)$, and two were not on any medications.

For the FMT procedure, the source of donor stool was OpenBiome stool bank in $60 \%$ (47/78), first degree relative in $36 \%(28 / 78)$, two cases from friends of the patient, and one from a medical student. Methods of delivery included mainly colonoscopy to terminal ileum 79.5\% (62/78), cecum 7.7\% $(6 / 78)$, and spray throughout the colon in one case. Three cases were delivered through gastrostomy or jejunostomy tube, four were performed via flexible sigmoidoscopy, one case via enteroscopy, and one by enema administration. The amount of stool used was $250 \mathrm{~mL}$ in 59\% (46/78) and $500 \mathrm{~mL}$ in $36 \%(28 / 78)$, and the remaining three cases had the following amounts used (480, 500 and $1200 \mathrm{~mL})$.

\section{Post-FMT data}

The median follow-up time was 25 months (interquartile range: 16 - 37 months). The primary cure rate for the whole study population was $76.9 \%(60 / 78)$. The early recurrence rate was $10.25 \%(8 / 78)$ where late recurrence was $12.8 \%$ (10/78). Out of the 18 recurrent cases, seven required repeat FMT where the remaining 11 responded to antibiotics. The secondary cure rate was (78/78) $100 \%$ at the end of the study (Fig. 1).

For the IBD subgroup, there was no statistically significant difference in the primary and secondary cure rates comparing patients with and without IBD. Also, the early and late recurrence rates as well as the percentage of patient requiring repeat FMT were comparable between the two groups. Table 2 summarizes these results.

Interestingly, after FMT 50\% (11/21) of IBD patients required some type of escalation of therapy that includes: change in class of medication $36 \%(4 / 11)$, addition of a different class of medication $27 \%(3 / 11)$ or surgery in $36 \%(4 / 11)$.

Regarding subjective report of IBD disease activity and symptoms after FMT, 28\% (6/21) reported improvement, 66\% $(14 / 21)$ reported no change and one patient said his disease activity became worse, although that patient didn't require any escalation of therapy.

Abdominal pain after FMT in IBD patient resolved in $33.3 \%(5 / 15)$ patients, improved in $13 \%(2 / 15)$ and remained the same in $53 \%(8 / 15)$. Diarrhea was reported in $61 \%(13 / 21)$ patients.

\section{Safety and adverse events}

New diagnoses after FMT were reported in eight patients included; one skin basal cell carcinoma, one dysphagia and Schatzki ring, one community-acquired pneumonia, one partial large bowel obstruction, one shingles and carpel tunnel syndrome and one patient required hospitalization for colonic decompression 2 years after the FMT. One patient reported a small amount of weight gain. One patient reported more sensitivity to gas-provoking foods such as broccoli and Brussels sprouts. Two patients noted improvement in their energy and overall well being. Interestingly, $75 \%$ said they would want FMT as their first option if CDI happened to recur and the rest 


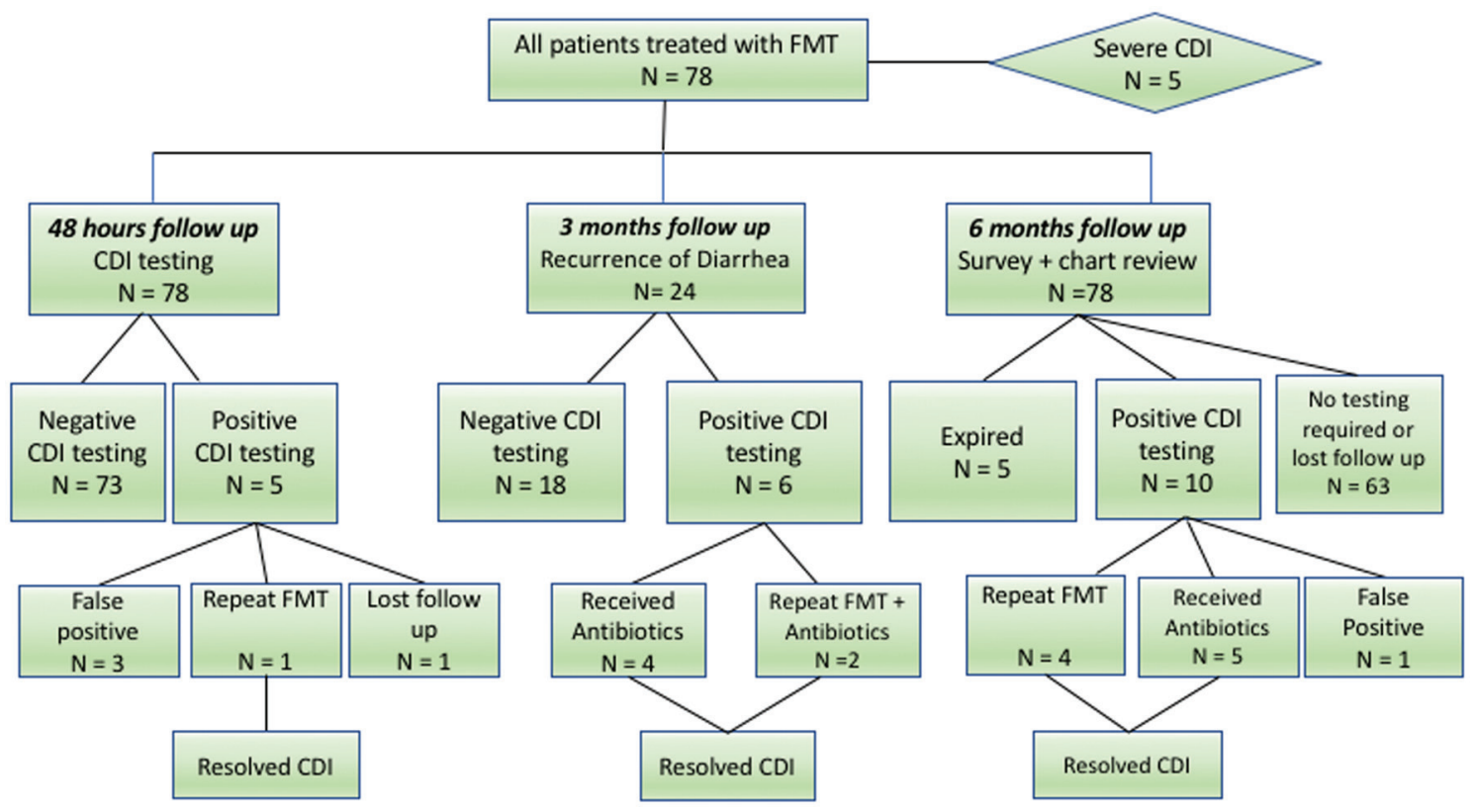

Figure 1. Flow diagram depicting all patients undergone FMT and their outcomes.

preferred discussion with their doctor prior to taking further steps.

For IBD patients, reported adverse events were IBD flare in five patients, and timeline of these hospitalizations were variable from 1 week to 6 months after FMT, with one of these hospitalized patients eventually requiring ileal resection. Two patients required colectomy and one patient required diverting ileostomy (originally had IPPA pouch) due to worsening of disease activity. Table 3 summarizes complications in IBD patients.

\section{Discussion}

In our long-term follow-up survey of 25 months to assess the safety and efficacy profile of FMT in 78 patients with non-severe CDI, a $78 \%$ and $71 \%$ cure rate after first FMT was found in non-IBD and IBD patients respectively and a combined primary cure rate of $76 \%$, reaching $100 \%$ in both populations after the second FMT or antibiotic therapy, with no reported serious adverse events directly related to FMT. These findings continue to demonstrate FMT as a favorable option for patients with recurrent and refractory $\mathrm{CDI}$ in the general population as well as in IBD patients.

Table 2. Comparison of Patients With IBD vs. Non-IBD

\begin{tabular}{llll} 
& IBD $(\mathbf{n}=\mathbf{2 1})$ & Non-IBD $(\mathbf{n}=\mathbf{5 7})$ & Total \\
\hline Primary cure & $15(71.4 \%)$ & $45(78.9 \%)$ & $60(76.9 \%)$ \\
Secondary cure & $21(100 \%)$ & $57(100 \%)$ & $78(100 \%)$ \\
Early recurrence & $2(9.5 \%)$ & $6(10.5 \%)$ & $8(10.25 \%)$ \\
Late recurrence & $4(19.0 \%)$ & $6(10.5 \%)$ & $10(12.8 \%)$ \\
Repeat FMT & $4(57.1 \%)$ & $3(42.8 \%)$ & $7(8.9 \%)$ \\
\hline
\end{tabular}

The short-term efficacy of FMT has already been established and well-studied with cure rates up to $90 \%$ in the current literature. More attention has been focused on the reliability and safety profile in long-term outcomes, especially in high risk populations such as the elderly, immunosuppressed and patients with IBD. Recent published guidelines by IDSA recommend treatment of refractory and recurrent CDI unresponsive to appropriate antibiotic therapy after the second recurrence in the general population [18]. No guidelines have however been established to guide FMT therapy for CDI in IBD patients, who pose a great challenge in treatment of recurrent CDI, with their immunosuppressed status, chronic illness and multiple hospitalizations. Part of the reason for lack of such guidelines could be attributed to the mixed data and debate on applications of FMT in treatment of IBD itself [22, 23 ] and reports of exacerbation of IBD and inducing flare-ups of disease in patients who were otherwise in remission [19].

The secondary cure rates on long-term FMT follow-up observed in our study are comparable to those reported by Brandt et al [24], where 77 patients with a mean follow-up duration of 17 months, resulted in a $98 \%$ secondary cure rate. Their primary cure rate was $91 \%$, compared to our findings of $79 \%$ in the total population. This difference could be attributed partially to a higher prevalence of IBD patients, although there was no statistically significant difference between our IBD and nonIBD primary cure rates. It is likely that due to the small sample size in both studies, methodological differences in delivery of stool, stool source and amount as well as other unmeasured factors such as comorbidities.

A large series published recently by Fischer et al in patients treated with FMT for recurrent/refractory CDI with concomitant IBD showed a cure rate of $79 \%$ after single FMT and up to $88 \%$ after two FMTs, which is also consistent with the results of our study [21]. The significant difference noted between 
Table 3. Complications After FMT in IBD Patients by Recurrence of CDI

\begin{tabular}{llll}
\hline & Recurrence $(\mathbf{n}=\mathbf{6})$ & No recurrence $(\mathbf{n}=\mathbf{1 5})$ & All IBD patients $(\mathbf{n}=\mathbf{2 1})$ \\
\hline Colectomy & $1(16.7)$ & $1(6.7)$ & $2(9.5)$ \\
Hospitalizations for IBD flare & $1(16.7)$ & $4(26.7)$ & $5(23.8)$ \\
Ileal resection & $1(16.7)$ & $0(0.0)$ & $1(4.7)$ \\
None & $3(50.0)$ & $10(66.7)$ & $13(61.9)$ \\
\hline
\end{tabular}

primary and secondary cure in most studies may be because the first FMT might not be sufficient to completely restore the gut microbiota diversity, yet still provide enough diversity to help it become more responsive to the use of antibiotics in treatment of future recurrences. Although the decision on repeating FMT immediately versus trying a course of antibiotics was not standardized and was managed on a case by case basis, given the unique presentations and complexity of disease in these patients, it would be helpful in future studies to try establishing randomized studies comparing the second FMT and a course of antibiotics.

More than half of the patients with IBD reported no change in disease activity (66.7\%) and only one patient reported worsening of symptoms. Escalation of therapy was required in half of the cases. This should still be encouraging for eradication of recurrent CDI in these patients with the most effective measure, and yet to allow flexibility in choice of appropriate therapy, especially in a disease where younger populations compose a large portion, and in whom biologic agents would be preferable.

The timeline of early and late recurrence was similar in non-IBD patients, occurring $10.5 \%$ for both early and late recurrences. In contrast, IBD patients had nearly twice the number of late recurrences as compared to early recurrences $(9.5 \%$, $19 \%$ ) respectively. These findings were different from a recent retrospective study done by Meighani et al [25] which showed a lower number of late recurrences ( $>90$ days) of $20 \%$ compared to early recurrences of $25 \%$ in the IBD population. This study was however limited to a 90 -day follow-up, which does not enable the detection of later recurrences, as observed in our study up to more than 1 year, in one patient. Even though we report $100 \%$ cure rate after the second FMT, clinicians should still have a high suspicion in evaluating and treating hospitalized IBD patients for worsening diarrheal illness even if they have been cleared of CDI and remained symptom-free for an extended period, given the fact that most recurrence episodes $(4 / 21,19 \%)$ occurred $>3$ months after FMT. Therefore, we still recommend checking for CDI in all IBD patients as per ACG guidelines [15].

An interesting finding in the IBD population was that $93 \%$ of the non-recurrence group patients after the first FMT were taking 5-aminosalicylate acid products as part of their treatment regimen, compared to $33 \%$ of the recurrence group $(\mathrm{P}<$ $0.0035)$. Similar findings were seen in the study of Fischer et al [21], but did not reach clinical significance. This raises the question about potential role of 5-ASA products in decreasing the chance of recurrence of CDI. ASA products play an important role in treatment of induction and in maintenance therapy in mild and moderate disease. ASA works by inhibiting leukocyte chemotaxis and production of chemotactic eicosanoids and interleukin-8 $[26,27]$. It also protects the intestinal mucosa from oxidative stress injury caused by oxygen-derived free radicals [28]. A retrospective study done to identify risk factors for CDI in UC patients and outcomes found that 5-ASA treatment independently protected UC patients from colectomy. A study on CDI induced colitis in rat modules found that 5-ASA strongly inhibits acute $C$ difficile toxin A-induced colitis [29]. Further studies would be useful to examine the association between use of 5-ASA products and the incidence, recurrence and complications of CDI.

Our study had several limitations including the retrospective nature of study and the potential for recall bias. We encountered difficulty in reaching all patients via the phone and there were also limitations pertinent to the use of chart review, which sometimes had missing data. Moreover, the antibiotic treatment protocol prescribed prior to FMT was not standardized. Results could also be affected by the diversities in the patient population with variations in comorbidities and reasons for FMT. Finally, there is a limitation from an absence of correlation with endoscopic findings in both IBD and non-IBD patients.

In conclusion, our positive long-term experience with FMT in the treatment of recurrent CDI, in a population having concomitant IBD, further supports the move towards early consideration of FMT. Even though our findings do not suggest that course of IBD is either altered or changed, they nevertheless support planned escalation of IBD therapy after eradication of CDI in patients with advanced disease.

\section{Author Contributions}

Obada M. Tabbaa: generation, collection, assembly, analysis and/or interpretation of data; drafting and revision of the manuscript. Mohammed M. Aboelsoud: drafting of the manuscript. Mark M. Mattar: conception and design of the study; generation, collection, assembly, analysis and/or interpretation of data; critical revision of the manuscript.

\section{Disclosures}

No financial support or grants were used to conduct this study. The manuscript was presented in part at the Crohn's and Colitis Congress Meeting in Las Vegas on January 19, 2018.

\section{References}

1. Lessa FC, Mu Y, Bamberg WM, Beldavs ZG, Dumyati 
GK, Dunn JR, Farley MM, et al. Burden of Clostridium difficile infection in the United States. N Engl J Med. 2015;372(9):825-834.

2. Borody TJ, Campbell J. Fecal microbiota transplantation: techniques, applications, and issues. Gastroenterol Clin North Am. 2012;41(4):781-803.

3. Seekatz AM, Aas J, Gessert CE, Rubin TA, Saman DM, Bakken JS, Young VB. Recovery of the gut microbiome following fecal microbiota transplantation. MBio. 2014;5(3):e00893-00814.

4. Fekety R, McFarland LV, Surawicz CM, Greenberg RN, Elmer GW, Mulligan ME. Recurrent Clostridium difficile diarrhea: characteristics of and risk factors for patients enrolled in a prospective, randomized, double-blinded trial. Clin Infect Dis. 1997;24(3):324-333.

5. McFarland LV, Surawicz CM, Rubin M, Fekety R, Elmer GW, Greenberg RN. Recurrent Clostridium difficile disease: epidemiology and clinical characteristics. Infect Control Hosp Epidemiol. 1999;20(1):43-50.

6. Redelings MD, Sorvillo F, Mascola L. Increase in Clostridium difficile-related mortality rates, United States, 1999-2004. Emerg Infect Dis. 2007;13(9):14171419.

7. Kelly CR, Ihunnah C, Fischer M, Khoruts A, Surawicz C, Afzali A, Aroniadis O, et al. Fecal microbiota transplant for treatment of Clostridium difficile infection in immunocompromised patients. Am J Gastroenterol. 2014;109(7):1065-1071.

8. Ricciardi R, Ogilvie JW, Jr., Roberts PL, Marcello PW, Concannon TW, Baxter NN. Epidemiology of Clostridium difficile colitis in hospitalized patients with inflammatory bowel diseases. Dis Colon Rectum. 2009;52(1):4045.

9. Nitzan O, Elias M, Chazan B, Raz R, Saliba W. Clostridium difficile and inflammatory bowel disease: role in pathogenesis and implications in treatment. World J Gastroenterol. 2013;19(43):7577-7585.

10. Sartor RB. Genetics and environmental interactions shape the intestinal microbiome to promote inflammatory bowel disease versus mucosal homeostasis. Gastroenterology. 2010;139(6):1816-1819.

11. Sokol H, Pigneur B, Watterlot L, Lakhdari O, Bermudez-Humaran LG, Gratadoux JJ, Blugeon S, et al. Faecalibacterium prausnitzii is an anti-inflammatory commensal bacterium identified by gut microbiota analysis of Crohn disease patients. Proc Natl Acad Sci U S A. 2008;105(43):16731-16736.

12. Wang W, Chen L, Zhou R, Wang X, Song L, Huang S, Wang $G$, et al. Increased proportions of Bifidobacterium and the Lactobacillus group and loss of butyrate-producing bacteria in inflammatory bowel disease. J Clin Microbiol. 2014;52(2):398-406.

13. Freeman HJ. Recent developments on the role of Clostridium difficile in inflammatory bowel disease. World J Gastroenterol. 2008;14(18):2794-2796.

14. Ananthakrishnan AN, Oxford EC, Nguyen DD, Sauk J, Yajnik V, Xavier RJ. Genetic risk factors for Clostridium difficile infection in ulcerative colitis. Aliment Pharmacol Ther. 2013;38(5):522-530.
15. Surawicz CM, Brandt LJ, Binion DG, Ananthakrishnan AN, Curry SR, Gilligan PH, McFarland LV, et al. Guidelines for diagnosis, treatment, and prevention of Clostridium difficile infections. Am J Gastroenterol. 2013;108(4):478-498; quiz 499.

16. Bakken JS, Borody T, Brandt LJ, Brill JV, Demarco DC, Franzos MA, Kelly C, et al. Treating Clostridium difficile infection with fecal microbiota transplantation. Clin Gastroenterol Hepatol. 2011;9(12):1044-1049.

17. Berg AM, Kelly CP, Farraye FA. Clostridium difficile infection in the inflammatory bowel disease patient. Inflamm Bowel Dis. 2013;19(1):194-204.

18. McDonald LC, Gerding DN, Johnson S, Bakken JS, Carroll $\mathrm{KC}$, Coffin SE, Dubberke ER, et al. Clinical practice guidelines for clostridium difficile infection in adults and children: 2017 update by the Infectious Diseases Society of America (IDSA) and Society for Healthcare Epidemiology of America (SHEA). Clin Infect Dis. 2018;66(7):987-994.

19. Khoruts A, Rank KM, Newman KM, Viskocil K, Vaughn BP, Hamilton MJ, Sadowsky MJ. Inflammatory bowel disease affects the outcome of fecal microbiota transplantation for recurrent clostridium difficile infection. Clin Gastroenterol Hepatol. 2016;14(10):1433-1438.

20. De Leon LM, Watson JB, Kelly CR. Transient flare of ulcerative colitis after fecal microbiota transplantation for recurrent Clostridium difficile infection. Clin Gastroenterol Hepatol. 2013;11(8):1036-1038.

21. Fischer M, Kao D, Kelly C, Kuchipudi A, Jafri SM, Blumenkehl M, Rex D, et al. Fecal microbiota transplantation is safe and efficacious for recurrent or refractory clostridium difficile infection in patients with inflammatory bowel disease. Inflamm Bowel Dis. 2016;22(10):2402-2409.

22. Moayyedi P, Surette MG, Kim PT, Libertucci J, Wolfe M, Onischi C, Armstrong D, et al. Fecal microbiota transplantation induces remission in patients with active ulcerative colitis in a randomized controlled trial. Gastroenterology. 2015;149(1):102-109 e106.

23. Rossen NG, Fuentes S, van der Spek MJ, Tijssen JG, Hartman JH, Duflou A, Lowenberg M, et al. Findings from a randomized controlled trial of fecal transplantation for patients with ulcerative colitis. Gastroenterology. 2015;149(1):110-118 e114.

24. Brandt LJ, Aroniadis OC, Mellow M, Kanatzar A, Kelly C, Park T, Stollman N, et al. Long-term follow-up of colonoscopic fecal microbiota transplant for recurrent Clostridium difficile infection. Am J Gastroenterol. 2012;107(7):1079-1087.

25. Meighani A, Hart BR, Bourgi K, Miller N, John A, Ramesh M. Outcomes of fecal microbiota transplantation for clostridium difficile infection in patients with inflammatory bowel disease. Dig Dis Sci. 2017;62(10):2870-2875.

26. Nielsen $\mathrm{OH}$, Verspaget HW, Elmgreen J. Inhibition of intestinal macrophage chemotaxis to leukotriene B4 by sulphasalazine, olsalazine, and 5-aminosalicylic acid. Aliment Pharmacol Ther. 1988;2(3):203-211.

27. Subramanian S, Rhodes JM, Hart CA, Tam B, Roberts CL, Smith SL, Corkill JE, et al. Characterization of epithelial IL-8 response to inflammatory bowel disease mu- 
cosal E. coli and its inhibition by mesalamine. Inflamm Bowel Dis. 2008;14(2):162-175.

28. Dallegri F, Ottonello L, Ballestrero A, Bogliolo F, Ferrando F, Patrone F. Cytoprotection against neutrophil derived hypochlorous acid: a potential mechanism for the therapeutic action of 5-aminosalicylic acid in ulcerative colitis. Gut. 1990;31(2):184-186.

29. Vigna SR. 5-aminosalicylic acid inhibits acute clostridium difficile toxin a-induced colitis in rats. Int J Inflam. 2014;2014:389621. 